\title{
A General Seifert-Van Kampen Theorem for Algebraic Fundamental Groups
}

\author{
By \\ Jakob STIX*
}

\begin{abstract}
A Seifert-Van Kampen theorem describes the fundamental group of a space in terms of the fundamental groups of the constituents of a covering and the configuration of connected components of the covering. Here we provide the combinatorial part of such a theorem for the most general sort of coverings. Thus a Seifert-Van Kampen theorem is reduced to a purely geometric statement of effective descent.
\end{abstract}

\section{Introduction}

The problem of describing the fundamental group of a space $X$ in terms of the fundamental groups of the constituents $X_{i}$ of an open covering was addressed by Van Kampen [VK33] and Seifert [ST34] in a special case. Nowadays we call such a statement a Seifert-Van Kampen theorem.

More recent contributions treat more general coverings and use limits [We61] or groupoids [Br67], [BRS84] or both [Al75] to formulate and prove their results; but they essentially stick to coverings of open subsets of topological spaces.

In the algebraic context, we find in [Ma73], [St89] a Seifert-Van Kampen theorem for fibre products of rings. The account in [SGA 1] IX $\S 5$ treats étale fundamental groups of connected schemes but requires a common base point for the parts of the 'generalized open' covering. However, [SGA 1] uses the tautological proof that exploits the following fact. The fundamental group of a space is equivalently described by its category of covering spaces, henceforth called covers for better distinction with 'generalized open' coverings.

Communicated by S. Mochizuki. Received April 21, 2004. Revised July 22, 2005.

2000 Mathematics Subject Classification(s): 14F35, 14H30, $20 \mathrm{E} 18$.

* Mathematisches Institut, Universität Bonn, Beringstraße 1, 53115 Bonn, Germany. 
According to the tautological proof a Seifert-Van Kampen theorem relative to a covering $\left\{X_{i} \rightarrow X\right\}$ is obtained in three steps.

(1) Descent: On the level of spaces a glueing property for covers with respect to the given covering is established. In the topological context this glueing property is obvious for open coverings. In general, descent theory deals with nothing but this question of glueing, cf. [Gi64]. We will regard as a generalized covering $\left\{X_{i} \rightarrow X\right\}$ anything that allows for 'effective descent', meaning glueing works or more precisely an equivalence of the category of covers of $X$ with the category of descent data relative to the given covering, see $\S 4.1$ and $\S 5.1$. Therefore any generalized covering leads to a Seifert-Van Kampen theorem.

(2) Discretising: Descent data for categories of covers are described as locally constant systems on a 2-complex with group data, see $\S 2$. This step eliminates the reference to a space in the description of covers, cf. $\S 4.3$.

(3) Abstract Seifert-Van Kampen: The fundamental group of a 2complex with group data is given by an amalgamation formula in terms of the respective vertex groups and the configuration of the 2-complex, see $\S 3$. The main result is the following statement which is essentially identical to the classical topological one:

Corollary 3.3. The fundamental group of the 2-complex E with group data $(\mathscr{G}, \alpha)$ and maximal subtree $T$ in its 1 -skeleton $E_{\leq 1}$ has the structure of a pro-finite amalgam as follows.

$$
\left(\left(\underset{v \in E_{0}}{*} \mathscr{G}(v)\right) * \widehat{\pi}_{1}\left(E_{\leq 1}, T\right)\right) /\langle\langle e d g e, \text { cocycle }\rangle) \stackrel{\sim}{\longrightarrow} \pi_{1}(E, \mathscr{G} ; T)
$$

where 'edge, cocycle' denote the homotopy relations as in Section 3.2.

The situation above is summarized in a diagram with notations to be explained later.

$$
\begin{aligned}
& \operatorname{Rev}(X) \stackrel{\cong}{\longrightarrow} \mathrm{DD}\left(T_{\bullet}, \operatorname{Rev}\right) \stackrel{\cong}{\longrightarrow} \operatorname{Ics}(E,(\mathscr{G}, \alpha))
\end{aligned}
$$

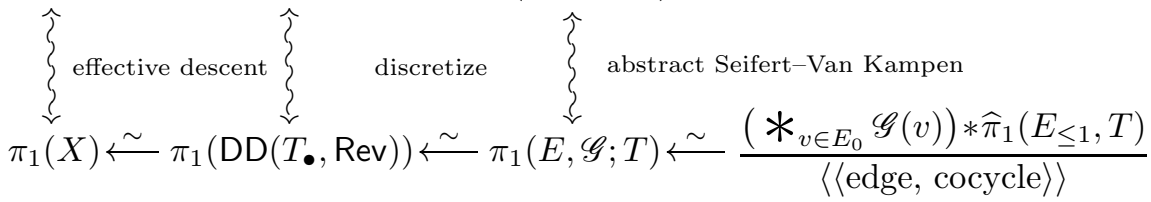

The purpose of this note is to accomplish with an exercise left to the reader in a footnote of [SGA 1] IX $\S 5$ which asks for a generalization of the treatment there. We achieve the combinatorial part for a general Seifert-Van Kampen 
theorem, thus reducing such an assertion to a purely geometric statement of effective descent. In our discussion 2-simplices appear due to the more general covering configurations that we allow. These lead to additional relations and a bigger combinatorial complexity.

The focus in this note will be algebraic fundamental groups. Hence all group theory deals with pro-finite groups. The categories of covers are Galois categories, cf. [SGA 1] V $\S 4$ and $\S 1$ below. But with appropriate modifications the treatment remains valid for arbitrary categories of covers, thus including the classical case of fundamental groups of topological spaces which admit universal covers. For more abstract but similar efforts with groupoids and toposes see [Le79], [Zo02]. In $\S 5$ we give some examples and applications.

This note arose from chapter 2 of the author's dissertation [Sx02] where the Seifert-Van Kampen theorem was established and applied in the context of logarithmic fundamental groups.

I would like to express my gratitude to Hiroaki Nakamura for an invitation to Okayama and the opportunity to deliver a talk on the subject, and to Eike Lau for a stimulating discussion about the contents of [Se80] back in 1999. The author acknowledges the support provided through the European Community's Human Potential Program under contract HPRN-CT-2000-00114, GTEM.

\section{$\S 1$. Categories of Covers}

For the convenience of the reader we recall the concept of a Galois category and its properties as in [SGA 1] $\mathrm{V} \S 4$.

Definition 1.1. (1) A Galois category is a category $\mathscr{C}$ such that the following list of axioms hold.

(G1) In $\mathscr{C}$ all finite projective limits exist. In particular there is a final object and fibre products exist.

(G2) In $\mathscr{C}$ all finite coproducts exist, as well as categorical quotients by actions of finite groups of automorphisms. In particular there is an initial object.

(G3) Any morphism $f$ of $\mathscr{C}$ admits a factorization $f=\iota \circ p$, where $\iota$ is the inclusion of a direct summand and $p$ is a strict epimorphism, i.e., $p$ is a morphism $X^{\prime} \rightarrow X$, such that with the projections $\mathrm{pr}_{i}: X^{\prime} \times_{X} X^{\prime} \rightarrow X$ for $i=1,2$ the following holds for all objects $T$ :

$$
\operatorname{Hom}_{\mathscr{C}}(X, T)=\left\{f^{\prime} \in \operatorname{Hom}_{\mathscr{C}}\left(X^{\prime}, T\right) \mid f^{\prime} \circ \operatorname{pr}_{1}=f^{\prime \prime} \circ \operatorname{pr}_{2}\right\},
$$

where the map is induced by $h \mapsto h \circ p$. 
Furthermore, $\mathscr{C}$ admits a fibre functor, which is any functor from $\mathscr{C}$ to the category sets of finite sets that

(G4) commutes with finite projective limits,

(G5) commutes with finite coproducts and quotients by finite groups, and maps strict epimorphisms to strict epimorphisms (i.e. surjective maps),

(G6) and which is conservative, i.e., if for a morphism $\varphi$ of $\mathscr{C}$ the map of sets $F(\varphi)$ is bijective, then $\varphi$ is an isomorphism.

(2) For any fibre functor $F$ of a Galois category $\mathscr{C}$ the group of automorphisms of $F$ is called the fundamental group of $\mathscr{C}$ with base point $F$ and is denoted by $\pi_{1}(\mathscr{C}, F)$.

The fundamental group $\pi_{1}(\mathscr{C}, F)$ is in a natural way a pro-finite group. A basis of open subgroups for the pro-finite topology is given by the kernels of the natural maps $\pi_{1}(\mathscr{C}, F) \rightarrow \operatorname{Aut}(F(X))$ that evaluate an automorphism at a specific connected object $X$ of $\mathscr{C}$. An object of $\mathscr{C}$ is called connected if it does not admit a nontrivial coproduct decomposition. An object $X$ is connected if and only if $\pi_{1}(\mathscr{C}, F)$ acts transitively on $F(X)$.

A fibre functor $F: \mathscr{C} \rightarrow$ sets enhances tautologically to a functor, still denoted $F$, from $\mathscr{C}$ with values in the category of finite, discrete, continuous $\pi_{1}(\mathscr{C}, F)$-sets. The following theorem of abstract Galois theory holds.

Theorem 1.2. $\quad$ Let $\mathscr{C}$ be a Galois category with fibre functors $F, F^{\prime}$.

(1) The functor $F: \mathscr{C} \rightarrow \pi_{1}(\mathscr{C}, F)$-sets is an equivalence of categories.

(2) The set $\pi_{1}\left(\mathscr{C}, F, F^{\prime}\right):=\operatorname{Isom}\left(F, F^{\prime}\right)$ of Tannaka paths from $F$ to $F^{\prime}$ is a pro-finite set and naturally a continuous bitorsor under the action of $\pi_{1}(\mathscr{C}, F)$ from the right and $\pi_{1}\left(\mathscr{C}, F^{\prime}\right)$ from the left.

(3) The groups $\pi_{1}(\mathscr{C}, F)$ and $\pi_{1}\left(\mathscr{C}, F^{\prime}\right)$ are canonically isomorphic up to composition with an inner automorphism.

Sketch of proof. First one constructs Galois hulls: any connected object $X \in \mathscr{C}$ is covered by a Galois object, i.e., a connected object $Y \rightarrow X$ such that \# $\operatorname{Aut}_{\mathscr{C}}(Y)=\# F(Y)$, or equivalently $Y / \operatorname{Aut}_{\mathscr{C}}(Y)$ is the final object of $\mathscr{C}$. Then one rigidifies the situation by choosing an element $y \in F(Y)$ for every Galois object $Y$. Between two thus pointed Galois objects $Y^{\prime}, Y$ there exists at most one morphism compatible with the pointing. If $Y^{\prime} \rightarrow Y$ exist, then using 
the pointings, $\operatorname{Aut}_{\mathscr{C}}(Y)$ is canonically a quotient of $\operatorname{Aut}_{\mathscr{C}}\left(Y^{\prime}\right)$. Consequently, the pointed Galois objects form a filtered directed system $I$, and $\pi_{1}(\mathscr{C}, F)$ is canonically isomorphic to the pro-finite group $\lim _{(Y, y) \in I} \operatorname{Aut} \mathscr{C}(Y)^{\mathrm{opp}}$. For details see [SGA 1] V 4.1, 5.2.

Definition 1.3. A functor between Galois categories is called exact if it satisfies the properties (G4) and (G5) above.

Theorem 1.4. Let $\mathscr{C}$ and $\mathscr{C}^{\prime}$ be Galois categories with fibre functors $F$ and $F^{\prime}$.

(1) Exact functors $H: \mathscr{C}^{\prime} \rightarrow \mathscr{C}$ up to natural isomorphism correspond uniquely to classes of continuous homomorphisms $\pi_{1} H: \pi_{1}(\mathscr{C}, F) \rightarrow \pi_{1}\left(\mathscr{C}^{\prime}, F^{\prime}\right)$ in the reverse direction up to composition with inner automorphisms.

(2) Let $H: \mathscr{C} \rightarrow \mathscr{C}^{\prime}$ be an exact functor between Galois categories. Then the following are equivalent.

(a) $\pi_{1} H$ is surjective.

(b) For any connected object $X^{\prime}$ of $\mathscr{C}^{\prime}$ the object $H\left(X^{\prime}\right)$ remains connected.

(c) $H$ is fully faithful.

Proof. [SGA 1] V 6.2, 6.9.

Example 1.5. Let $X$ be a connected, locally path connected topological space that admits a universal covering space. Let $\operatorname{cov}(X)$ be the category of covering spaces ('covers' in the terminology of this note) of $X$ with finite fibres. To any point $x \in X$ we obtain a fibre functor $F_{x}$ that is 'taking the fibre above $x$ ' such that

$$
\pi_{1}\left(\operatorname{cov}(X), F_{x}\right)=\left(\widehat{\pi}_{1}(X, x)\right)^{\text {opp }},
$$

where $\widehat{\pi}_{1}(X, x)$ denotes the pro-finte completion of the fundamental group of homotopy classes of loops based at $x$ and the superscript 'opp' denotes the opposite group where the order of composition has been reversed. The order reversion is necessary because topological paths act naturally from the right on fibres while the Tannaka paths act from the left.

Example 1.6. Let $X$ be a locally noetherian, connected scheme. Then the category $\operatorname{Rev}_{X}$ of all finite étale covers of $X$ is a Galois category. A fibre functor can be constructed from any geometric point $x \in X$ by taking the appropriate fibre, because $\operatorname{Rev}_{x}$ is canonically equivalent to the category of finite sets. The resulting fundamental group is the étale fundamental group

$$
\pi_{1}(X, x) .
$$




\section{§2. Combinatorial Covers of 2-Complexes with Group Data}

This section owes much to [SGA 1] IX $\S 5$. See also [Se80] for the discrete situation without 2-simplices, and [We61] for the topological situation.

\section{$\S 2.1 . \quad 2-c o m p l e x e s$}

The underlying combinatorics of descent data is simplicial, but truncated in degree 2. Degeneration maps are not necessary and are thus omitted.

The simplicial category truncated in degree $2, \Delta_{\leq \mathbf{2}}$, consists of objects $[n]:=\{0, \ldots, n\}$ for $n=0,1,2$ and has strictly monotone maps as morphisms. There are face maps $\partial_{i}:[n-1] \rightarrow[n]$ for $0 \leq i \leq n$ which omit the value $i$. The set $\operatorname{Hom}([0],[n])$ of vertices of the $n$-simplex $[n]$ consists of maps $v_{i}$ with image $i$.

The category of 2-complexes in a category $\mathscr{C}$ is the category of contravariant functors $T_{\bullet}: \Delta_{\leq 2} \rightarrow \mathscr{C}$. The value $T_{\bullet}([n])=: T_{n}$ is called the object in degree $n$ or the $n$ simplices of $T_{\bullet}$. The value of $\partial_{i}$ is called the $i^{\text {th }}$ boundary map. Essentially $T_{\bullet}$ is a diagram in $\mathscr{C}$

$$
T_{0} \leftleftarrows T_{1} \leftleftarrows T_{2}
$$

and $T_{0}, T_{1}, T_{2}$ are referred to as vertices, edges and faces respectively.

\section{$\S 2.2$. Group data on a 2-complex of sets}

By a 2-complex $E$ we mean a 2-complex of sets in the sense of the previous section. One may consider $E$ as a small category: its objects are the elements of the $E_{n}$ for $n=0,1,2$ and its morphisms are formally obtained as $\partial: s \rightarrow t$ where $s \in E_{n}$ and $t=E(\partial)(s)$.

Let $\Delta_{n}=\left\{\sum_{i=0}^{n} \lambda_{i} e_{i} \in \mathbb{R}_{\geq 0}^{n+1} \mid \sum_{i} \lambda_{i}=1\right\}$ denote the topological $n$ simplex. Then we have a well known topological realization functor $|E|=$ $\coprod E_{n} \times \Delta_{n} / \sim$. Here $\sim$ identifies $(s, d(x))$ with $(E(\partial)(s), x)$ for all $\partial:[m] \rightarrow[n]$, its corresponding linear map $d: \Delta_{m} \rightarrow \Delta_{n}$ sending $e_{i}$ to $e_{\partial(i)}$, and $s \in E_{n}$ and $x \in \Delta_{m}$. We call $E$ connected if $|E|$ is a connected topological space.

Definition 2.1. $\quad$ Group data $(\mathscr{G}, \alpha)$ on the 2-complex $E$ consists of the following.

(i) A mapping $\mathscr{G}$ from the category $E$ to the category of pro-finite groups: to a simplex $s \in E_{n}$ is attributed a pro-finite group $\mathscr{G}(s)$ and to a map $\partial: s \rightarrow t$ is attached a morphism $\mathscr{G}(\partial): \mathscr{G}(s) \rightarrow \mathscr{G}(t)$. 
(ii) For every 2-simplex (vef) of the barycentric subdivision $\left(v \in E_{0}, e \in\right.$ $E_{1}, f \in E_{2}$ and boundary maps $\partial^{\prime}, \partial$ such that $\left.\partial^{\prime}(f)=e, \partial(e)=v\right)$ we fix an element $\alpha_{v e f} \in \mathscr{G}(v)$ such that the following diagram commutes:

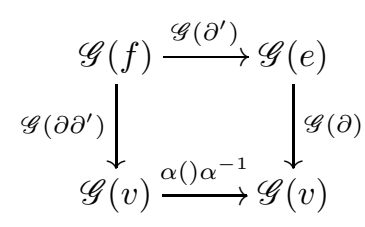

The group data $(\mathscr{G}, \alpha)$ simply describes a functor from $E$ to the category of pro-finite groups with outer morphisms in terms of genuine homomorphisms of pro-finite groups. For an essentially identical notion in the context of discrete groups but with injective maps $\mathscr{G}(\partial)$ see [Co92] 'labeled 2-complexes of groups'.

\section{§2.3. Locally constant systems}

Let $M$ be a $G$-set and $f: H \rightarrow G$ a group homomorphism. We refer to the induced $H$-set structure on $M$ as $f^{*} M$.

Definition 2.2. Let $(\mathscr{G}, \alpha)$ be group data on the 2-complex $E$. A $(\mathscr{G}, \alpha)$-system $M$ on $E$ consists of the following:

(i) for every simplex $s \in E$ a finite discrete continuous $\mathscr{G}(s)$-set $M_{s}$,

(ii) for every $\partial: s \rightarrow t$ a $\mathscr{G}(s)$-equivariant boundary map $m_{\partial}: M_{s} \rightarrow \mathscr{G}(\partial)^{*} M_{t}$, such that

(iii) for every 2-simplex (vef) of the barycentric subdivision with $\partial^{\prime}(f)=$ $e, \partial(e)=v$ the following commutes.

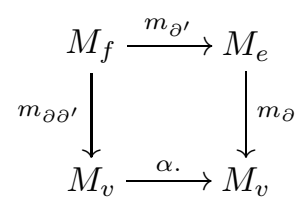

Diagram (2.2) is a little inaccurate in that it does not record the group action. The map $\left(\alpha\right.$.), action by $\alpha$, is merely a $\mathscr{G}(v)$-equivariant map $M_{v} \rightarrow$ $\left(\alpha() \alpha^{-1}\right)^{*} M_{v}$, because $\alpha \gamma m=\left(\alpha \gamma \alpha^{-1}\right) \alpha m$. Thus modified, diagram (2.2) is $\mathscr{G}(f)$-equivariant as $\mathscr{G}\left(\partial^{\prime}\right) * \mathscr{G}(\partial)^{*}=\mathscr{G}\left(\partial \partial^{\prime}\right)^{*}\left(\alpha() \alpha^{-1}\right)^{*}$ by $(2.1)$.

Morphisms of $(\mathscr{G}, \alpha)$-systems on $E$ are defined in the obvious way: a collection of $\mathscr{G}(s)$-equivariant maps that commute with the $m$ 's. 
Definition 2.3. A $\quad(\mathscr{G}, \alpha)$-system $\mathrm{M}$ on $E$ is called locally constant if all of its boundary maps $m_{\partial}$ are bijective. The category of all locally constant systems on $E$ is denoted by $\operatorname{lcs}(E,(\mathscr{G}, \alpha))$.

Proposition 2.4. Let $(E,(\mathscr{G}, \alpha))$ be a connected 2-complex with group data. Then its category of locally constant systems $\operatorname{Ics}(E,(\mathscr{G}, \alpha))$ is a Galois category.

Proof. We need to check the axioms (G1)-(G6) of section $\S 1$. Fibre products, final object, coproducts, initial object, quotients by finite group actions and the factorization condition on maps are constructed 'simplex wise' by the respective property in the Galois categories of $\mathscr{G}(s)$-sets. This works as the pullback functors $\mathscr{G}(\partial)$ respect these properties.

It remains to construct a fibre functor. For any simplex $s$ the evaluation $F_{s}: \operatorname{Ics}(E,(\mathscr{G}, \alpha)) \rightarrow$ sets which maps $M$ to the underlying set of $M_{s}$ yields a fibre functor. This follows as the categorical constructions above are performed 'simplex wise'. Furthermore $F_{s}$ is conservative because $|E|$ is connected.

Corollary 2.5. For every simplex $s \in E$

$$
F_{s}: \operatorname{lcs}(E,(\mathscr{G}, \alpha)) \rightarrow \mathscr{G}(s) \text {-sets, } \quad M \mapsto M_{s}
$$

is a canonical exact functor of Galois categories.

\section{§2.4. The topological component}

Let $E$ be a 2-complex. There is an associated oriented graph $E_{\leq 1}$ with vertices $E_{0}$ and edges $E_{1}$ such that $\partial_{0}$ (resp. $\partial_{1}$ ) map an edge to its target (resp. origin). Again we denote its topological realization by $\left|E_{\leq 1}\right|$.

For $M \in \operatorname{Ics}(E,(\mathscr{G}, \alpha))$ we construct an oriented graph $M_{\leq 1}$ over $E_{\leq 1}$ as follows. The vertices of $M_{\leq 1}$ are $\coprod_{v \in E_{0}} M_{v}$ and $\coprod_{e \in E_{1}} M_{e}$ serves as the set of edges. The target/origin maps are induced by the $m_{\partial}$ and the map $M_{\leq 1} \rightarrow E_{\leq 1}$ is the obvious one.

This assignment is certainly functorial. Its composition with the topological realization functor $|\cdot|$ is denoted by $|\cdot \leq 1|$ and yields a finite cover $\left|M_{\leq 1}\right| \rightarrow\left|E_{\leq 1}\right|$ of topological spaces.

Let $\operatorname{cov}(X)$ be the category of finite topological covers of a topological space $X$. Then the following is immediate from the simplexwise construction of products, coproducts and quotients.

Proposition 2.6. The functor $|\cdot \leq 1|: \operatorname{lcs}(E,(\mathscr{G}, \alpha)) \rightarrow \operatorname{cov}\left(\left|E_{\leq 1}\right|\right)$ is an exact functor between Galois categories. 
As usual, to a maximal subtree $T \subset E_{\leq 1}$ is attributed a fibre functor $F_{T}: \operatorname{cov}\left(\left|E_{\leq 1}\right|\right) \rightarrow$ sets by assigning to a cover $p: X \rightarrow\left|E_{\leq 1}\right|$ the set of connected components $\pi_{0}\left(p^{-1}(|T|)\right)$. The resulting fundamental group $\pi_{1}\left(\operatorname{cov}\left(\left|E_{\leq 1}\right|\right), F_{T}\right)$ is isomorphic to the opposite group of the pro-finite completion of the topological fundamental group of $\left|E_{\leq 1}\right|$, see example 1.5. We obtain a canonical isomorphism

$$
\widehat{\pi}_{1}\left(E_{\leq 1}, T\right):=\widehat{\operatorname{Fr}}\left(E_{1}\right) /\langle\langle\vec{e} \mid e \in T\rangle\rangle \stackrel{\sim}{\longrightarrow} \pi_{1}\left(\operatorname{cov}\left(\left|E_{\leq 1}\right|\right), F_{T}\right)
$$

by sending the element $\vec{e}$ to the action

$$
\pi_{0}\left(p^{-1}(|T|)\right) \cong p^{-1}\left(\partial_{0}(e)\right) \cong \pi_{0}\left(p^{-1}(|e|)\right) \cong p^{-1}\left(\partial_{1}(e)\right) \cong \pi_{0}\left(p^{-1}(|T|)\right),
$$

where $|e| \subset\left|E_{\leq 1}\right|$ denotes the topological realization of the edge $e$ and all bijections are induced from the natural inclusions. Here $\widehat{\operatorname{Fr}}(S)$ denotes the free pro-finite group on a set $S, \vec{s}$ is the symbol for the generator corresponding to $s \in S$, and $\langle\langle I\rangle\rangle$ denotes the minimal normal closed subgroup containing $I$. We got rid of taking the opposite group here because the path $\vec{e}$, which originates in $\partial_{1}(e)$ and leads to $\partial_{0}(e)$, acts in the opposite direction.

Let $F_{T}$ also denote the fibre functor $F_{T}(|\cdot \leq 1|)$ on $\operatorname{Ics}(E,(\mathscr{G}, \alpha))$. For every simplex $s \in E_{0}$ the group $\mathscr{G}(s)$ canonically acts on $F_{T}(M)$ for $M \in$ $\operatorname{lcs}(E,(\mathscr{G}, \alpha))$ via $M_{s}=\pi_{0}\left(p^{-1}(s)\right)=\pi_{0}\left(p^{-1}(|T|)\right)$.

Let $*$ be the notation for pro-finite amalgamation, cf. [RZ00].

Proposition 2.7. The functor

$$
Q: \operatorname{lcs}(E,(\mathscr{G}, \alpha)) \rightarrow\left(\left(\underset{v \in E_{0}}{*} \mathscr{G}(v)\right) * \widehat{\pi}_{1}\left(E_{\leq 1}, T\right)\right) \text {-sets }
$$

encapsulating the above operations on $F_{T}(\cdot)$ is fully faithful.

Proof. We prove that $Q$ conserves connectedness of objects which amounts to transitivity of the group action. We argue by contradiction. Let $M \in$ $\operatorname{lcs}(E,(\mathscr{G}, \alpha))$ be connected and let $N \subset F_{T}(M)$ be a nonempty proper subset stable under the action of $\widehat{\pi}_{1}\left(E_{\leq 1}, T\right)$ and $\mathscr{G}(v)$ for $v \in E_{0}$. The stability under $\widehat{\pi}_{1}\left(E_{\leq 1}, T\right)$ implies that $N$ is respected by the boundary maps $m_{\partial}$, because $\vec{e}$ acts as $m_{\partial_{1}} m_{\partial_{0}}^{-1}$, and thus extends to a subgraph $N_{\leq 1} \subset M_{\leq 1}$.

We need to show that it extends further over 2-simplices. This is a local question that only depends on the simplices in the boundary of a given face $f \in E_{2}$. We define $N_{f}$ as the preimage under $m_{\partial}$ of $N_{s} \subset M_{s}$ for any $\partial: f \rightarrow s$ and need to verify that this subset of $M_{f}$ is well defined. It clearly suffices for any 2-simplex (vef) of the barycentric subdivision to compare $\partial^{\prime}: f \rightarrow e$ with 
the composition $\partial \partial^{\prime}: f \rightarrow e \rightarrow v$. But as $\alpha_{v e f} \in \mathscr{G}(v)$ respects $N_{v}$ we have by $(2.2)$

$$
m_{\partial \partial^{\prime}}^{-1}\left(N_{v}\right)=m_{\partial \partial^{\prime}}^{-1}\left((\alpha \cdot)^{-1}\left(N_{v}\right)\right)=m_{\partial^{\prime}}^{-1} m_{\partial}^{-1}\left(N_{v}\right)=m_{\partial^{\prime}}^{-1}\left(N_{e}\right)
$$

and thus $N$ originates from an object $N \in \operatorname{Ics}(E,(\mathscr{G}, \alpha))$ which is a subobject of $M$ and thus contradicts the connectedness of $M$.

\section{§3. The Abstract Seifert-Van Kampen Theorem}

The abstract Seifert-Van Kampen theorem provides an isomorphism of an amalgamated product of the group data along a 2-complex with the fundamental group of the respective category of locally constant systems.

\section{§3.1. $\mathscr{G}$-doted paths}

Let $(E,(\mathscr{G}, \alpha))$ be a connected 2-complex with group data. As above, $F_{v}$ and $F_{T}$ denote the respective fibre functors of $\operatorname{Ics}(E,(\mathscr{G}, \alpha))$ assigned to a vertex $v \in E_{0}$ and a maximal subtree $T \subset E_{\leq 1}$. The respective fundamental groups are abbreviated as $\pi_{1}(E, \mathscr{G} ; v)$ and $\pi_{1}(E, \mathscr{G} ; T)$.

Definition 3.1. A $\mathscr{G}$-doted path from $i \in E_{0}$ to $f \in E_{0}$ is a pro-word $\gamma$ in

$$
\left(\underset{v \in E_{0}}{*} \mathscr{G}(v)\right) * \widehat{\operatorname{Fr}}\left(E_{1}\right)
$$

such that successive letters are always geometrically adjacent and whose first (resp. last) letter is localized in $i$ (resp. $f$ ), i.e., at every finite level $\gamma$ is represented by a word such that consecutive letters consist in one of the following options:

(i) $\ldots \vec{e} \vec{\varepsilon} \ldots$ for $e, \varepsilon \in E_{1}$ such that $\partial_{0}(e)=\partial_{1}(\varepsilon)$,

(ii) $\ldots \vec{e} g \ldots$ for $e \in E_{1}$ and $g \in \mathscr{G}\left(\partial_{0}(e)\right)$,

(iii) $\ldots g \vec{e} \ldots$ for $e \in E_{1}$ and $g \in \mathscr{G}\left(\partial_{1}(e)\right)$,

(iv) $\ldots g g^{\prime} \ldots$ for $g, g^{\prime} \in \mathscr{G}(v)$ for some $v \in E_{0}$,

and its first letter is $g \in \mathscr{G}(i)$ or $\vec{e}$ corresponding to $e \in E_{1}$ with $\partial_{1}(e)=i$ (resp. its last letter is $g \in \mathscr{G}(f)$ or $\vec{e}$ corresponding to $e \in E_{1}$ with $\partial_{0}(e)=f$ ).

The subset of $\mathscr{G}$-doted paths in

$$
\left(\underset{v \in E_{0}}{*} \mathscr{G}(v)\right) * \widehat{\operatorname{Fr}}\left(E_{1}\right)
$$

is denoted by $\Omega(E, \mathscr{G} ; i, f)$. If $i=f=v$ we call them $\mathscr{G}$-doted loops based at $v$. They form a subgroup $\Omega(E, \mathscr{G} ; v)$. 


\section{§3.2. Homotopy relations}

Let $M \in \operatorname{Ics}(E,(\mathscr{G}, \alpha))$ be a locally constant system and $\partial: s \rightarrow t$ a boundary map in $E$.

The intuition behind the following is to interpret $m_{\partial}: M_{s} \rightarrow M_{t}$ as the continuation of fibres of a cover along a path $\vec{\partial}$ of the base which goes in the opposite direction, namely $\vec{\partial}: t \rightarrow s$. The map $m_{\partial}$ will be called the monodromy action along the path $\vec{\partial}$. These conventions allow to compose paths as usually and at the same time to act from the left on fibres. Composition of paths will lead to more maps between various $M_{s}$. Ultimately that leads to shorter and more natural expressions for the homotopy relations as these are governed by the combinatorics of paths.

The edge relation. Let $e \in E_{1}$ be an edge. We want to study $M$ locally at $e$ and define a path $\vec{e}=\vec{\partial}_{1}\left(\vec{\partial}_{0}\right)^{-1}: \partial_{1}(e) \rightarrow \partial_{0}(e)$

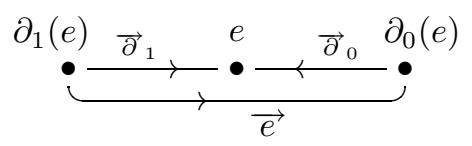

and understand that its monodromy action on fibres is given by

$$
m_{\partial_{1}} m_{\partial_{0}}^{-1}: M_{\partial_{0}(e)} \rightarrow M_{\partial_{1}(e)}
$$

As the $m_{\partial_{i}}$ are $\mathscr{G}(e)$ equivariant we obtain for $g \in \mathscr{G}(e)$ the relation

$$
\mathscr{G}\left(\partial_{1}\right)(g) m_{\partial_{1}} m_{\partial_{0}}^{-1}=m_{\partial_{1}} g m_{\partial_{0}}^{-1}=m_{\partial_{1}} m_{\partial_{0}}^{-1} \mathscr{G}\left(\partial_{0}\right)(g) .
$$

Hence for all $e \in E_{1}$ and $g \in \mathscr{G}(e)$ we have the edge relation of $\mathscr{G}$-doted paths from $\partial_{1}(e)$ to $\partial_{0}(e)$ :

$$
\mathscr{G}\left(\partial_{1}\right)(g) \vec{e}=\vec{e} \mathscr{G}\left(\partial_{0}\right)(g)
$$

The cocycle relation. The standard 2 -simplex $\Delta_{2}$ consists of

$$
\left(\Delta_{2}\right)_{0}=\{0,1,2\},\left(\Delta_{2}\right)_{1}=\{(01),(02),(12)\},\left(\Delta_{2}\right)_{2}=\{(012)\}
$$

and boundary maps $\partial_{i}$ that forget the $i^{\text {th }}$ entry. Let $f \in E_{2}$ be a face. The minimal simplicial subset $E(f) \subset E$ containing $f$ consists of all its boundaries $\partial(f)$ and equals the image of the unique map $\Delta_{2} \rightarrow E$ mapping (012) to $f$.

We want to study $M$ locally at $f$. The above reduces this to the study of its pullback $\left.M\right|_{\Delta_{2}}$ on the standard 2-simplex. We set $\alpha_{i j k}=\alpha_{i,(i j),(i j k)}$ for any 
permutation $\{i, j, k\}=\{0,1,2\}$ and also apply to it the interpretation of being a monodromy operator along the opposite direction of a path $\vec{\alpha}_{i j k}$ this time being a loop based at $(i)$. We obtain the following pictures of paths for $\Delta_{2}$
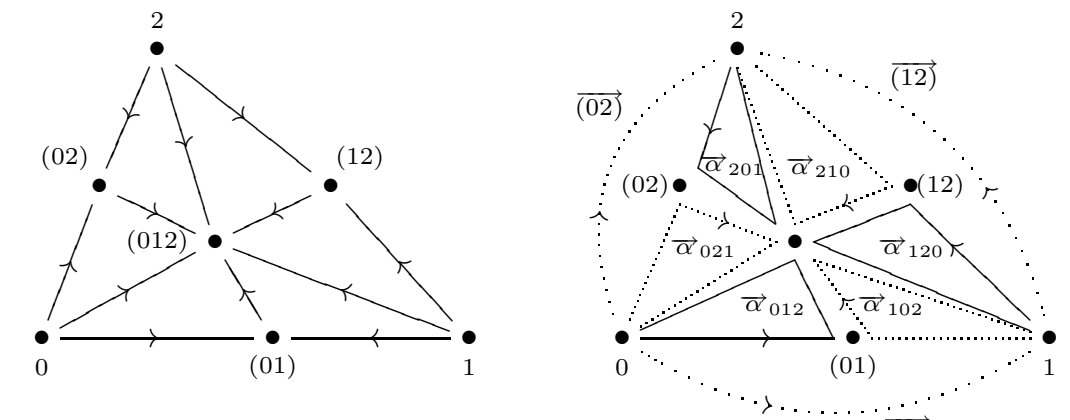

$\overrightarrow{(01)}$

where the loops $\vec{\alpha}_{i j k}$ turn around a face of the barycentric subdivision once by passing through the simplices in ascending order according to their degrees.

There are six relations that $M$ is required to satisfy on $E(f)$. For any permutation of $\{i, j, k\}=\{0,1,2\}$ we have

$$
\begin{gathered}
\alpha_{i j k} m_{v_{i}}=\left\{\begin{array}{l}
m_{\partial_{0}} m_{\partial_{k}} i>j \\
m_{\partial_{1}} m_{\partial_{k}} i<j
\end{array}\right. \\
\text { with }\left\{\begin{array}{l}
\partial_{0}:(i j) \rightarrow i \text { for } i>j \\
\partial_{1}:(i j) \rightarrow i \text { for } i<j
\end{array} \text { and } \partial_{k}:(i j k) \rightarrow(i j),\right.
\end{gathered}
$$

which holds as maps $M_{f} \rightarrow M_{v_{i}(f)}$ by the compatibility condition (2.2) of Definition 2.2.

In the interpretation of monodromy actions along paths relation (3.5) is due to composition of paths around the 2 -simplex $i(i j)(i j k)$ of the barycentric subdivision, as can be seen in (3.4).

The map $m_{\partial_{1}} m_{\partial_{0}}^{-1}: M_{\partial_{0} \partial_{k}(f)} \rightarrow M_{\partial_{1} \partial_{k}(f)}$ is the monodromy action of the path $\overrightarrow{\partial_{k} f}: \partial_{1}\left(\partial_{k} f\right) \rightarrow \partial_{0}\left(\partial_{k} f\right)$. By turning once around the barycentre of diagram (3.4) - thus incorporating all the six of the relations once - an easy calculation yields the cocycle relation (3.6) which is a relation of $\mathscr{G}$-doted loops based at $v_{0}(f)$ for any $f \in E_{2}$ :

$$
1=\left(\overrightarrow{\partial_{2} f}\right) \alpha_{102}\left(\alpha_{120}\right)^{-1}\left(\overrightarrow{\partial_{0} f}\right) \alpha_{210}\left(\alpha_{201}\right)^{-1}\left(\left(\overrightarrow{\partial_{1} f}\right)^{-1}\right) \alpha_{021}\left(\alpha_{012}\right)^{-1}
$$


The homotopy relations consist of the normal subgroup

$$
H \triangleleft\left(\underset{v \in E_{0}}{*} \mathscr{G}(v)\right) * \widehat{\operatorname{Fr}}\left(E_{1}\right)
$$

generated by the cocycle relation (3.6) and the edge relation (3.3) for all possible parameters:

$$
H=\left\langle\left\langle\begin{array}{c}
\mathscr{G}\left(\partial_{1}\right)(g) \vec{e}\left(\vec{e} \mathscr{G}\left(\partial_{0}\right)(g)\right)^{-1} \\
\left(\overrightarrow{\partial_{2} f}\right) \alpha_{102} \alpha_{120}{ }^{-1}\left(\overrightarrow{\partial_{0} f}\right) \alpha_{210} \alpha_{201}^{-1}\left(\left(\overrightarrow{\partial_{1} f}\right)\right)^{-1} \alpha_{021} \alpha_{012}{ }^{-1}
\end{array}\right\rangle .\right.
$$

The paths that show up in these relations are interpreted as the respective elements of $\widehat{\operatorname{Fr}}\left(E_{1}\right)$. By intersecting with the group $\Omega(E, \mathscr{G} ; v)$ of loops, we obtain the normal subgroup $H_{\Omega, v}$ of homotopy relations for loops based at $v$.

\section{$\S 3.3 . \quad$ The theorem}

Theorem 3.2 abstract Seifert-Van Kampen theorem. Let E be a connected 2-complex with group data $(\mathscr{G}, \alpha)$. With the above notation we have a commutative diagram that yields two descriptions of the fundamental group of $\operatorname{lcs}(E,(\mathscr{G}, \alpha))$.

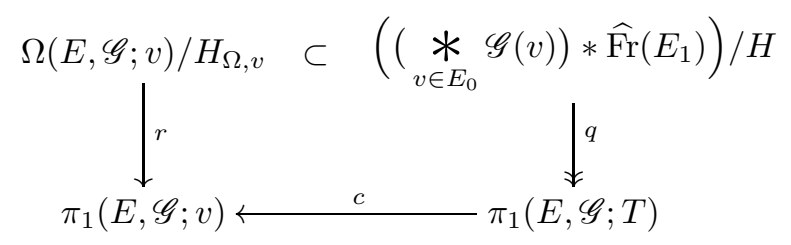

The morphisms c, q, r have the following properties:

(1) $c$ is the isomorphism between the two $\pi_{1}$ induced by the natural isomorphism $F_{v} \rightarrow F_{T}$ derived from $v \in T$,

(2) $q$ is induced by the functor $Q$ of Proposition 2.7, it is surjective and its kernel is generated as a normal subgroup by $\{\vec{e} \mid e \in T\}$,

(3) $r$ is an isomorphism which is induced by the canonical operation of $v$-based $\mathscr{G}$-doted loops on the fibre $F_{v}(\cdot)$.

Proof. (1) The assertion on $c$ is trivial. (2) Next we deal with $q$. As the kernel of $\widehat{\operatorname{Fr}}\left(E_{1}\right) \rightarrow \widehat{\pi}_{1}\left(E_{\leq 1}, T\right)$ is generated by the edges from $T$ we need to show that the functor $Q$ yields an isomorphism

$$
\left(\left(\underset{v \in E_{0}}{*} \mathscr{G}(v)\right) * \widehat{\pi}_{1}\left(E_{\leq 1}, T\right)\right) / \bar{H} \stackrel{\sim}{\longrightarrow} \pi_{1}(E, \mathscr{G} ; T)
$$


where $\bar{H}$ is the image of $H$. Equivalently we need to show that $Q$ induces an equivalence of $\operatorname{Ics}(E,(\mathscr{G}, \alpha))$ with the full subcategory of those objects from $\left(*_{v \in E_{0}} \mathscr{G}(v)\right) * \widehat{\pi}_{1}\left(E_{\leq 1}, T\right)$-sets on which $\bar{H}$ act trivially. By Proposition 2.7 the functor $Q$ is fully faithful and by the motivational arguments of $\S 3.2$ it is clear that $\bar{H}$ acts trivially on the essential image of $Q$.

Let $\tilde{M}$ be a $\left(\left(*_{v \in E_{0}} \mathscr{G}(v)\right) * \widehat{\pi}_{1}\left(E_{\leq 1}, T\right)\right)$-set on which $\bar{H}$ acts trivial. We need to show that it is isomorphic to a $Q(M)$ for an $M \in \operatorname{lcs}(E,(\mathscr{G}, \alpha))$. We build $M$ degree by degree. For $v \in E_{0}$ we set $M_{v}=\tilde{M}$ with the induced $\mathscr{G}(v)$ action. For $e \in E_{1}$ we set $M_{e}=\tilde{M}$ with the $\mathscr{G}(e)$ action induced by $\mathscr{G}\left(\partial_{0}\right): \mathscr{G}(e) \rightarrow \mathscr{G}\left(\partial_{0}(e)\right)$. For $\partial_{i}: e \rightarrow v$ from degree 1 to 0 we thus set $m_{\partial_{0}}=i d$ and $m_{\partial_{1}}=\vec{e}$ as element of $\widehat{\pi}_{1}\left(E_{\leq 1}, T\right)$ acting on $\tilde{M}$. The edge relations (3.3) imply that these $m_{\partial}$ 's are $\mathscr{G}(e)$ equivariant.

To extend this construction up to the 2-simplices $f \in E_{2}$ it suffices to work in the respective closures $E(f)$, or even over the standard 2-simplex $\Delta_{2}$, which we will do in the sequel. We set $M_{(012)}=\tilde{M}$ with $\mathscr{G}(012)$ action induced by $\mathscr{G}(012) \rightarrow \mathscr{G}(0)$. By the six relations (3.5) discussed above the maps $m_{\partial}$ for $\partial:(012) \rightarrow s$ for any simplex $s$ are uniquely determined: $m_{v_{0}}$ for the 'vertex' $v_{0}:(012) \rightarrow(0)$ is the identity on $\tilde{M}$; now solve for the various $m_{\delta}$ using the six relations by turning around the barycentre of the picture (3.4) of paths of $\Delta_{2}$. The consistency of the solutions is equivalent to the cocycle condition.

(3) Finally we treat $r$. It is defined by the action on $M_{v}$ via the following two operations. For an edge $e \in E_{1}$ the path $\vec{e} \in \widehat{\pi}_{1}\left(E_{\leq 1}, T\right)$ acts by

$$
m_{\partial_{1}} m_{\partial_{0}}^{-1}: M_{\partial_{0}(e)} \rightarrow M_{\partial_{1}(e)}
$$

For a vertex $v^{\prime}$ and $g \in \mathscr{G}\left(v^{\prime}\right)$ the path $g$ acts by the $\mathscr{G}\left(v^{\prime}\right)$ action on $M_{v^{\prime}}$. Note that this is possible due to the condition of letters in $\mathscr{G}$-doted paths to be geometrically adjacent. It yields a left action on $M_{v}$ as we start from the right by continuating in the direction opposite to the one of the path. This definition of $r$ obviously is compatible with $q$ proving that $H_{\Omega, v}$ acts trivial. This defines $r$ in a compatible way such that the diagram (3.7) commutes.

For $v_{1}, v_{2} \in E_{0}$, let $\gamma_{v_{1}, v_{2}}$ denote the finite geodesic from $v_{1}$ to $v_{2}$ in the tree $T$, i.e., the unique minimal connecting path in $T$ (this is a finite combinatorial path and not a pro-path). We treat it as an element of $\widehat{\operatorname{Fr}}\left(E_{1}\right)$ such that $\vec{e}=\gamma_{\partial_{1}(e), \partial_{0}(e)}$ and $(\vec{e})^{-1}=\gamma_{\partial_{0}(e), \partial_{1}(e)}$ and so on by composition.

Let $\rho: \Omega(E, \mathscr{G} ; v) / H_{\Omega, v} \rightarrow \operatorname{Aut}(M)$ be a continuous action on a finite discrete set $M$. For injectivity of $r$ it is sufficient to extend any $\rho$ to an action of $\left(\boldsymbol{*}_{v \in E_{0}} \mathscr{G}(v)\right) * \widehat{\operatorname{Fr}}\left(E_{1}\right)$ on $M$ such that $H$ and $e \in T$ act trivially. We define 
for $g \in \mathscr{G}\left(v^{\prime}\right)$ and $e \in E_{1}$

$$
\begin{aligned}
g & \mapsto \rho\left(\gamma_{v, v^{\prime}} g \gamma_{v, v^{\prime}}^{-1}\right): M \rightarrow M \\
\vec{e} & \mapsto \rho\left(\gamma_{v, \partial_{1}(e)} \vec{e} \gamma_{v, \partial_{0}(e)}^{-1}\right): M \rightarrow M
\end{aligned}
$$

which is possible as $\rho$ is only applied to $\mathscr{G}$-doted paths. This obviously extends the action to

$$
\rho:\left(\underset{v \in E_{0}}{*} \mathscr{G}(v)\right) * \widehat{\operatorname{Fr}}\left(E_{1}\right) \rightarrow \operatorname{Aut}(M)
$$

such that $H$ (homotopy relations are $\mathscr{G}$-doted paths) and $e \in T$ act trivially (due to the properties of geodesics in trees).

As a pro-finite group $\pi_{1}(E, \mathscr{G} ; T)$ is generated by the elements

$$
q(g)=q\left(\gamma_{v, v^{\prime}} g \gamma_{v, v^{\prime}}^{-1}\right), \quad q(\vec{e})=q\left(\gamma_{v, \partial_{1}(e)} \vec{e} \gamma_{v, \partial_{0}(e)}^{-1}\right)
$$

for $g \in \mathscr{G}\left(v^{\prime}\right)$ and $e \in E_{1}$ whence $r$ is surjective.

Corollary 3.3. In the situation of Theorem 3.2 we have a canonical isomorphism

$$
\left(\left(\underset{v \in E_{0}}{*} \mathscr{G}(v)\right) * \widehat{\pi}_{1}\left(E_{\leq 1}, T\right)\right) /\langle\langle\text { edge, cocycle }\rangle) \stackrel{\sim}{\longrightarrow} \pi_{1}(E, \mathscr{G} ; T)
$$

where 'edge, cocycle' denote the homotopy relations as above.

Remark. In the description of $\pi_{1}(E, \mathscr{G} ; T)$ the groups $\mathscr{G}(f)$ for faces $f \in E_{2}$ don't show up. This corresponds to the fact that triple intersections of open parts of a generalized open covering are only necessary to formulate the cocycle condition for the glueing morphisms.

\section{$\S 4$. Discretising Descent Data}

\section{$\S 4.1$. Descent data}

Let $T_{\bullet}$ be a 2 -complex in the category $\mathscr{C}$ and let $\mathscr{F} \rightarrow \mathscr{C}$ be a category fibred over $\mathscr{C}$, cf. [SGA 1] VI, with $\mathscr{F}(S)$ as category of sections above the object $S$.

Definition 4.1. The category $\operatorname{DD}\left(T_{\bullet}, \mathscr{F}\right)$ of descent data for $\mathscr{F} / \mathscr{C}$ relative $T_{\bullet}$ has as objects pairs $\left(X^{\prime}, \varphi\right)$ where $X^{\prime} \in \mathscr{F}\left(T_{0}\right)$ and $\varphi$ is an isomorphism $\partial_{0}^{*} X^{\prime} \stackrel{\sim}{\longrightarrow} \partial_{1}^{*} X^{\prime}$ in $\mathscr{F}\left(T_{1}\right)$ such that the cocycle condition holds, i.e., 
the following commutes in $\mathscr{F}\left(T_{2}\right)$ :

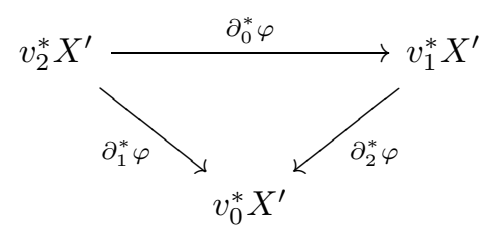

Morphisms $f:\left(X^{\prime}, \varphi\right) \rightarrow\left(Y^{\prime}, \psi\right)$ in $\operatorname{DD}\left(T_{\bullet}, \mathscr{F}\right)$ are morphisms $f: X^{\prime} \rightarrow Y^{\prime}$ in $\mathscr{F}\left(T_{0}\right)$ such that its two pullbacks $\partial_{0}^{*} f$ and $\partial_{1}^{*} f$ are compatible with $\varphi, \psi$, i.e., $\partial_{1}^{*} f \circ \varphi=\psi \circ \partial_{0}^{*} f$.

Our main example is the following. Let $h: S^{\prime} \rightarrow S$ be a map of schemes. There is an associated 2-complex of schemes

$$
S_{\bullet}(h): \quad S^{\prime} \leftleftarrows S^{\prime} \times_{S} S^{\prime} \leftleftarrows S^{\prime} \times_{S} S^{\prime} \times_{S} S^{\prime}
$$

The fibre products are with respect to $h$ and the value of $\partial_{i}$ is the projection under omission of the $i^{\text {th }}$ component. We abbreviate $\operatorname{DD}(S \bullet(h), \mathscr{F})$ by $\mathrm{DD}(h, \mathscr{F})$.

\section{$\S 4.2$. Ordered descent data}

Definition 4.2. A map $f: X \rightarrow Y$ (of schemes) is called a monomorphism if the relative diagonal $X \rightarrow X \times_{Y} X$ is an isomorphism. Equivalently, it defines an injective map $X(T) \hookrightarrow Y(T)$ on $T$ valued points for arbitrary $T$.

Let $h=\coprod h_{i}: S^{\prime}=\coprod_{i \in I} S_{i}^{\prime} \rightarrow S$ be a map of schemes and $\prec$ an ordering on the set of indices $I$. Let $S_{\bullet}^{\prec}(h) \subset S_{\bullet}(h)$ be the open and closed sub-2complex of schemes of ordered partial products

$$
S_{0}^{\prec}(h)=S^{\prime}, S_{1}^{\prec}(h)=\coprod_{i_{0} \prec i_{1}} S_{i_{0}}^{\prime} \times{ }_{S} S_{i_{1}}^{\prime}, S_{2}^{\prec}(h)=\coprod_{i_{0} \prec i_{1} \prec i_{2}} S_{i_{0}}^{\prime} \times_{S} S_{i_{1}}^{\prime} \times{ }_{S} S_{i_{2}}^{\prime}
$$

If $S$ is locally noetherian then in particular $S=\coprod S_{i}$ where $\left\{S_{i}\right\}=\pi_{0}(S)$ is the set of connected components. We get an example of a map for the following proposition if we take $S^{\prime}=\coprod S_{i}$ and $h_{i}$ is the respective closed immersion $S_{i} \hookrightarrow S$.

Proposition 4.3. Let $h_{i}: S_{i}^{\prime} \rightarrow S$ be monomorphisms, let $h$ be the map $\coprod h_{i}: S^{\prime}=\coprod S_{i}^{\prime} \rightarrow S$ and choose an ordering $\prec$ on the set of indices. Then the 
natural open and closed immersion $j: S_{\bullet}^{\prec}(h) \hookrightarrow S_{\bullet}(h)$ induces an equivalence of categories of descent data

$$
j^{*}: \mathrm{DD}(h, \mathscr{F}) \stackrel{\cong}{\mathrm{DD}}\left(S_{\bullet}^{\prec}(h), \mathscr{F}\right) .
$$

Proof. Let $S_{i j}^{\prime \prime}=S_{i}^{\prime} \times{ }_{S} S_{j}^{\prime}$ and $S_{i j k}^{\prime \prime \prime}=S_{i}^{\prime} \times_{S} S_{j}^{\prime} \times_{S} S_{k}^{\prime}$. The "glueing" isomorphisms $\varphi$ of descent data decomposes as $\varphi=\coprod_{i, j} \varphi_{i j}\left(\operatorname{resp} . \varphi^{\prec}=\coprod_{i \prec j} \varphi_{i j}\right)$ according to the decomposition $S_{2}(f)=\coprod_{i, j} S_{i j}^{\prime \prime}\left(\right.$ resp. $\left.S_{2}^{\prec}(f)=\coprod_{i \prec j} S_{i j}^{\prime \prime}\right)$.

Due to $h_{i}$ being monomorphic we have $S_{i i i}^{\prime \prime \prime}=S_{i}^{\prime}$ and $S_{i j i}^{\prime \prime \prime}=S_{i j}^{\prime \prime}$. For these indices the cocycle condition (4.10) yields $\varphi_{i i} \circ \varphi_{i i}=\varphi_{i i}$ and $\varphi_{j i} \circ \varphi_{i j}=\varphi_{i i}$. Hence $\varphi_{i i}=\mathrm{id}$ and $\varphi_{j i}=\varphi_{i j}^{-1}$. Thus $j^{*}$ in (4.11) is an equivalence, because $\varphi$ and $\varphi^{\prec}$ carry the same information.

\section{§4.3. Discretisation}

By discretising descent data we mean to construct a 2-complex with group data and an equivalence of the category of descent data with the associated category of locally constant systems.

In what follows we require our fibred category $\mathscr{F} \rightarrow \mathscr{C}$ to be fibred in Galois categories. More precisely, we have a notion of connected objects in $\mathscr{C}$ and any $T \in \mathscr{C}$ is a coproduct of connected components. Over connected objects $\mathscr{F}$ takes values in Galois categories and pullback functors are exact. For example, we may choose any reasonable geometric category for $\mathscr{C}$ and assume that $\mathscr{F}$ be a suitable category of covers.

If $X \in \mathscr{F}(S)$ and $T \subset S$ is a connected component we refer to the restriction of $X$ on $T$ as $\left.X\right|_{T}$.

Let $T_{\bullet}$ be a 2-complex in $\mathscr{C}$. Let $E=\pi_{0}\left(T_{\bullet}\right)$ be its 2-complex of connected components: the 2-complex in sets built by degree-wise application of the connected component functor. For a simplex $s \in E$ we fix a base point in $s$, i.e., a fibre functor $F_{s}$ of $\mathscr{F}(s)$. To a boundary map $\partial: s \rightarrow s^{\prime}$ we fix a choice of a path in the sense of [SGA 1] V, namely $\vec{\partial}: F_{s^{\prime}} \rightarrow T(\partial) F_{s}$ corresponding to a transformation $\vec{\partial}: F_{s} \circ T(\partial)^{*} \rightarrow F_{s^{\prime}}$.

For a 2-simplex (vef) of the barycentric subdivision with $\partial^{\prime}: f \rightarrow e$ and $\partial: e \rightarrow v$ we obtain a loop

$$
\alpha_{v e f}:=\vec{\partial} \overrightarrow{\partial^{\prime}}\left(\overrightarrow{\partial \partial^{\prime}}\right)^{-1} \in \pi_{1}\left(v, F_{v}\right)
$$

that is in the fundamental group of the component $v \subset T_{0}$ (actually $T(\partial) \overrightarrow{\partial^{\prime}}$ instead of $\overrightarrow{\partial^{\prime}}$ is correct). We define the group data $(\mathscr{G}, \alpha)$ on $E$ as follows: 
$\mathscr{G}(s)=\pi_{1}\left(s, F_{s}\right)$ for any simplex $s \in E$ and to $\partial: s \rightarrow s^{\prime}$ is associated

$$
\mathscr{G}(\partial): \pi_{1}\left(s, F_{s}\right) \stackrel{\pi_{1}(T(\partial))}{\longrightarrow} \pi_{1}\left(s^{\prime}, T(\partial) F_{s}\right) \stackrel{\vec{\partial}() \vec{\partial}^{-1}}{\longrightarrow} \pi_{1}\left(s^{\prime}, F_{s^{\prime}}\right) .
$$

The elements $\alpha$ is defined as in (4.12) above.

Proposition 4.4. The fibre functors $F_{s}$ and paths $\vec{\partial}$ as above fix a functor

$$
\operatorname{disc}: \operatorname{DD}\left(T_{\bullet}, \mathscr{F}\right) \rightarrow \operatorname{Ics}(E,(\mathscr{G}, \alpha))
$$

which is an equivalence of Galois categories.

Proof. Let $\left(X^{\prime}, \varphi\right)$ be a descent datum relative $T_{\bullet}$. We define $\operatorname{disc}\left(X^{\prime}, \varphi\right)=$ $M$ as follows.

$$
\begin{array}{llc}
v \in E_{0} & M_{v}=F_{v}\left(\left.X^{\prime}\right|_{v}\right) & \text { as } \pi_{1}\left(v, F_{v}\right) \text {-sets, } \\
e \in E_{1} \rightsquigarrow M_{e}=F_{e}\left(\left.T\left(\partial_{0}\right)^{*} X^{\prime}\right|_{e}\right) & \text { as } \pi_{1}\left(e, F_{e}\right) \text {-sets, } \\
f \in E_{2} \rightsquigarrow M_{f}=F_{f}\left(\left.T\left(\partial_{0} \partial_{0}\right)^{*} X^{\prime}\right|_{f}\right) \text { as } \pi_{1}\left(f, F_{f}\right) \text {-sets. }
\end{array}
$$

We define boundary maps $m_{\partial}$ for $\partial: e \rightarrow v$ with $e \in E_{1}$ as

$$
\begin{aligned}
& m_{\partial_{1}}: F_{e}\left(\left.T\left(\partial_{0}\right)^{*} X^{\prime}\right|_{e}\right) \stackrel{\left.\varphi\right|_{e}}{\longrightarrow} F_{e}\left(\left.T\left(\partial_{1}\right)^{*} X^{\prime}\right|_{e}\right) \stackrel{\vec{\partial}_{1}}{\longrightarrow} F_{\partial_{1}(e)}\left(\left.X^{\prime}\right|_{\partial_{1}(e)}\right) \\
& m_{\partial_{0}}: F_{e}\left(\left.T\left(\partial_{0}\right)^{*} X^{\prime}\right|_{e}\right) \stackrel{\vec{\partial}_{0}}{\longrightarrow} F_{\partial_{0}(e)}\left(\left.X^{\prime}\right|_{\partial_{0}(e)}\right) .
\end{aligned}
$$

Recall from $\S 2.1$ that the map [0] $\rightarrow[2]$ with image $i$ was called $v_{i}$. We define boundary maps $m_{\partial}$ starting from the face $f \in E_{2}$ as:

$$
\begin{aligned}
& m_{v_{0}}: F_{f}\left(\left.T\left(v_{2}\right)^{*} X^{\prime}\right|_{f}\right) \stackrel{\left.\partial_{1}^{*} \varphi\right|_{f}}{\longrightarrow} F_{f}\left(\left.T\left(v_{0}\right)^{*} X^{\prime}\right|_{f}\right) \stackrel{\vec{v}_{0}}{\longrightarrow} F_{v_{0}(f)}\left(\left.X^{\prime}\right|_{v_{0}(f)}\right) \\
& m_{\partial_{1}}: F_{f}\left(\left.T\left(v_{2}\right)^{*} X^{\prime}\right|_{f}\right) \stackrel{\vec{\partial}_{1}}{\longrightarrow} F_{\partial_{1}(f)}\left(\left.T\left(\partial_{0}\right)^{*} X^{\prime}\right|_{\partial_{1}(f)}\right) \\
& m_{v_{2}}: F_{f}\left(\left.T\left(v_{2}\right)^{*} X^{\prime}\right|_{f}\right) \stackrel{\vec{v}_{2}}{\longrightarrow} F_{v_{2}(f)}\left(\left.X^{\prime}\right|_{v_{2}(f)}\right) \\
& m_{\partial_{0}}: F_{f}\left(\left.T\left(v_{2}\right)^{*} X^{\prime}\right|_{f}\right) \stackrel{\vec{\partial}_{0}}{\longrightarrow} F_{\partial_{0}(f)}\left(\left.T\left(\partial_{0}\right)^{*} X^{\prime}\right|_{\partial_{0}(f)}\right) \\
& m_{v_{1}}: F_{f}\left(\left.T\left(v_{2}\right)^{*} X^{\prime}\right|_{f}\right) \stackrel{\left.\partial_{0}^{*} \varphi\right|_{f}}{\longrightarrow} F_{f}\left(\left.T\left(v_{1}\right)^{*} X^{\prime}\right|_{f}\right) \stackrel{\vec{v}_{1}}{\longrightarrow} F_{v_{1}(f)}\left(\left.X^{\prime}\right|_{v_{1}(f)}\right) \\
& m_{\partial_{2}}: F_{f}\left(\left.T\left(v_{2}\right)^{*} X^{\prime}\right|_{f}\right) \stackrel{\left.\partial_{0}^{*} \varphi\right|_{f}}{\longrightarrow} F_{f}\left(\left.T\left(v_{1}\right)^{*} X^{\prime}\right|_{f}\right) \stackrel{\vec{\partial}_{2}}{\longrightarrow} F_{\partial_{2}(f)}\left(\left.T\left(\partial_{0}\right)^{*} X^{\prime}\right|_{\partial_{2}(f)}\right)
\end{aligned}
$$

The boundary maps $m_{\partial}$ thus defined obey condition (2.2) of Definition 2.2 for all six possible choices: for their effect on fibres the following identity

$$
\partial_{i}^{*} \varphi:=T\left(\partial_{i}\right)^{*} \varphi=\left(\vec{\partial}_{i}\right)^{-1} \varphi \vec{\partial}_{i}
$$


holds proving condition (2.2) in five cases. It remains to check (2.2) for the 2-simplex $\left(v_{0}(f), \partial_{2}(f), f\right)$ of the barycentric subdivision, namely to verify

$$
m_{\partial_{1}} m_{\partial_{2}}=\alpha_{\left(v_{0}(f), \partial_{2}(f), f\right)} m_{v_{0}} .
$$

This amounts by the definition of the various $m_{\partial}$ to

$$
\left(\vec{\partial}_{1} \varphi\right)\left(\vec{\partial}_{2} \partial_{0}^{*} \varphi\right)=\left(\vec{\partial}_{1} \vec{\partial}_{2}\left(\vec{v}_{0}\right)^{-1}\right)\left(\vec{v}_{0} \partial_{1}^{*} \varphi\right)
$$

Using (4.13) the relation (4.14) is equivalent to

$$
\partial_{2}^{*} \varphi \partial_{0}^{*} \varphi=\partial_{1}^{*} \varphi
$$

which agrees with the cocycle condition (4.10) on $\varphi$ from descent theory.

The assignment $\left(X^{\prime}, \varphi\right) \mapsto \operatorname{disc}\left(X^{\prime}, \varphi\right)$ is clearly functorial. Hence we have defined a functor. To define an inverse let $M \in \operatorname{Ics}(E,(\mathscr{G}, \alpha))$ and determine $X^{\prime} \in \mathscr{F}\left(T_{0}\right)=\prod_{v \in E_{0}} \mathscr{F}(v)$ as $\left.X^{\prime}\right|_{v}$ corresponding to $M_{v}$ for all $v \in E_{0}$. The boundary maps from edges to vertices define a map $\varphi: T\left(\partial_{0}\right)^{*} X^{\prime} \rightarrow T\left(\partial_{1}\right)^{*} X^{\prime}$ and the cocycle condition holds for this $\left(X^{\prime}, \varphi\right)$ just by reversing the above argument that proved that disc yields locally constant systems.

\section{$\S 5 . \quad$ Examples and Applications}

\section{§5.1. Effective descent}

Let us assume that $\mathscr{C}$ has fibre products such that for a morphism $h$ the 2-complex $S_{\bullet}(h)$ in $\mathscr{C}$ as in $\S 4.1$ is defined. Let $\mathscr{F}$ be fibred over $\mathscr{C}$.

Definition 5.1. In the above context $h: S^{\prime} \rightarrow S$ is called a faithful (resp. descent, or even effective descent) morphism for $\mathscr{F}$ if $h^{*}$ is a faithful functor (resp. fully faithful functor, or even an equivalence of categories) between $\mathscr{F}(S)$ and $\operatorname{DD}(h, \mathscr{F})$. The adjective universal may be added as usual in case the respective property prevails to hold after arbitrary base extension.

\section{§5.2. Examples from étale covers of schemes}

Let $\mathscr{F}$ be the category Rev of finite étale covers of schemes. From [SGA 1] we have the following theorem.

Theorem 5.2. Let the map of schemes $h: S^{\prime} \rightarrow S$ satisfy one of the following properties: 
(1) $h$ is proper, surjective and of finite presentation,

(2) $h$ is fppf, i.e., faithfully flat and of finite presentation,

(3) $h$ is fpqc, i.e., faithfully flat and quasi-compact.

Then $h$ is a universal effective descent morphism for finite étale covers, i.e., for Rev.

\section{Proof. IX 4.12, IX 4.9 and IX 4.1 [SGA 1].}

Corollary 5.3. Let $h: S^{\prime} \rightarrow S$ be an effective descent morphism for Rev, e.g., satisfies one of the conditions of Theorem 5.2. Assume that $S$ is connected and $S, S^{\prime}$ are locally noetherian. Let $S^{\prime}=\coprod S_{v}^{\prime}$ be the decomposition into connected components. Let $\bar{s}$ be a geometric point of $S$, let $\bar{s}(t)$ be a geometric point of the simplex $t \in \pi_{0}\left(S_{\bullet}(h)\right)$, and let $T$ be a maximal tree in the graph $\Gamma=\pi_{0}\left(S_{\bullet}(h)\right)_{\leq 1}$. For every boundary map $\partial: t \rightarrow t^{\prime}$ let $\gamma_{t^{\prime}, t}$ : $\bar{s}\left(t^{\prime}\right) \rightarrow S_{\bullet}(h)(\partial) \bar{s}(t)$ be a fixed path in the sense of algebraic paths between base points. Then canonically with respect to all these choices

$$
\pi_{1}(S, \bar{s}) \cong\left(\left(\underset{v \in \pi_{0}\left(S^{\prime}\right)}{*} \pi_{1}\left(S_{v}^{\prime}, \bar{s}(v)\right)\right) * \widehat{\pi}_{1}(\Gamma, T)\right) / H
$$

where $H$ is the normal subgroup generated by the cocycle and edge relations

$$
\begin{gathered}
\pi_{1}\left(\partial_{1}\right)(g) \vec{e}=\vec{e} \pi_{1}\left(\partial_{0}\right)(g) \\
\left(\overrightarrow{\partial_{2} f}\right) \alpha_{102}^{(f)}\left(\alpha_{120}^{(f)}\right)^{-1}\left(\overrightarrow{\partial_{0} f}\right) \alpha_{210}^{(f)}\left(\alpha_{201}^{(f)}\right)^{-1}\left(\overrightarrow{\partial_{1} f}\right)^{-1} \alpha_{021}^{(f)}\left(\alpha_{012}^{(f)}\right)^{-1}=1 \\
\hline
\end{gathered}
$$

for all parameter values $e \in S_{1}(h), g \in \pi_{1}(e, \bar{s}(e))$, and $f \in S_{2}(h)$. The map $\pi_{1}\left(\partial_{i}\right)$ uses the fixed path $\gamma_{\partial_{i}(e), e}$ and finally $\alpha_{i j k}^{(f)}$ is defined using $v \in S_{0}(h)$ and $e \in S_{1}(h)$ determined by $v_{i}(f)=v,\left\{\partial_{0}(e), \partial_{1}(e)\right\}=\left\{v_{i}(f), v_{j}(f)\right\}$ as

$$
\alpha_{i j k}^{(f)}=\gamma_{v, e} \gamma_{e, f}\left(\gamma_{v, f}\right)^{-1} \in \pi_{1}(v, \bar{s}(v)) .
$$

Proof. Definition 5.1, Proposition 4.4, Theorem 3.2. Compare also with IX Theorem 5.1 [SGA 1].

Corollary 5.4. Let $h: S^{\prime} \rightarrow S$ be an effective descent morphism for Rev. Assume that $S$ is connected and $S, S^{\prime}$ are locally noetherian. Let $S^{\prime}=$ $\coprod_{v} S_{v}^{\prime}$ be the decomposition into connected components and assume that $\left.h\right|_{S_{v}^{\prime}}$ : $S_{v}^{\prime} \rightarrow S$ is a monomorphism. 
With the analogous choices of base points and paths connecting them as above and with a maximal tree $T$ in the graph $\Gamma=\pi_{0}\left(S_{\bullet}^{\prec}(h)\right)_{\leq 1}$ we have canonically with respect to all these choices an isomorphism

$$
\pi_{1}(S, \bar{s}) \cong\left(\left(\underset{v \in \pi_{0}\left(S^{\prime}\right)}{*} \pi_{1}\left(S_{v}^{\prime}, \bar{s}(v)\right)\right) * \widehat{\pi}_{1}(\Gamma, T)\right) / H
$$

where $H$ is the normal subgroup generated by the cocycle and edge relations for the respective group data on $S_{\bullet}^{\prec}(h)$.

Proof. In addition to the above we involve also Proposition 4.3.

Example 5.5. $\quad$ Let $\coprod h_{v}: \coprod S_{v}^{\prime} \rightarrow S$ be an effective descent morphism for Rev composed of monomorphisms $h_{i}$. Assume moreover that every 1-simplex is simply connected and $\left(S_{\bullet}^{\prec}\left(\coprod h_{v}\right)\right)_{2}$ is empty. This holds, for example, in the case of a semistable curve over an algebraically closed field with smooth irreducible components with respect to the covering by the disjoint union of its irreducible components. Let $\Gamma$ be the graph $\left(\pi_{0}\left(S_{\bullet}^{\prec}\left(\coprod h_{v}\right)\right)\right)_{\leq 1}$ as above which in the case of a semistable curve is just its dual graph. Then

$$
\pi_{1}(S, \bar{s}) \cong\left(\underset{v \in \pi_{0}\left(S^{\prime}\right)}{*} \pi_{1}\left(S_{v}^{\prime}, \bar{s}(v)\right)\right) * \widehat{\pi}_{1}(\Gamma, T) .
$$

Example 5.6. Let $h: S^{\prime} \rightarrow S$ be an effective descent morphism for Rev such that $S^{\prime}$ is connected and simply connected. Let $E=\pi_{0}\left(S_{\bullet}(h)\right)$ be the corresponding 2-complex. The edge relation holds for trivial reasons and the cocycle relation becomes the path homotopy relation for the combinatorial fundamental group of the simplicial space $E$. Let $T \subset E$ be a maximal subtree. Then

$$
\pi_{1}(S, \bar{s}) \cong \widehat{\pi}_{1}(E, T) .
$$

These assumptions are satisfied for a reduced, irreducible, proper, but singular curve over an algebraically closed field $k$ which is birational to $\mathbb{P}_{k}^{1}$ with respect to its normalization map. If $X$ is semistable with a single component whose normalization has genus 0 then

$$
\pi_{1}(X) \cong \widehat{\operatorname{Fr}}(\operatorname{Sing}(X)(k))
$$

is the free pro-finite group on the set of singular points.

\section{§5.3. Examples from number theory}

Theorem 5.2 may also be applied to number theoretic questions. 
Example 5.7. Let $L / K$ be a Galois extension of algebraic number fields with Galois group $G$ and $\mathfrak{o}_{L}, \mathfrak{o}_{K}$ the respective rings of integers. Then the natural map $\operatorname{Spec}\left(\mathfrak{o}_{L}\right) \rightarrow \operatorname{Spec}\left(\mathfrak{o}_{K}\right)$ is an effective descent morphism for Rev. Let $S^{\prime \prime}=\operatorname{Spec}\left(\mathfrak{o}_{L} \otimes_{\mathfrak{o}_{K}} \mathfrak{o}_{L}\right)$ which has $\# G$ irreducible components:

$$
\coprod_{G} \operatorname{Spec}\left(\mathfrak{o}_{L}\right) \stackrel{(i d, g)}{\longrightarrow} S^{\prime \prime}
$$

Connectedness of $S^{\prime \prime}$ is equivalent to the inertia subgroups generate $G$ which we will assume for the sequel, e.g., $\pi_{1}\left(\mathfrak{o}_{K}\right)=1$ or even $K=\mathbb{Q}$.

Then $\operatorname{Spec}\left(\mathfrak{o}_{L} \otimes_{\mathfrak{o}_{K}} \mathfrak{o}_{L} \otimes_{\mathfrak{o}_{K}} \mathfrak{o}_{L}\right)$ is connected as well and the topological part of the Seifert-Van Kampen formula vanishes. If we select the base points on the diagonals then we may choose all $\alpha=1$. Thus

$$
\pi_{1}\left(\mathfrak{o}_{L} \otimes_{\mathfrak{o}_{K}} \mathfrak{o}_{L}\right) \longrightarrow \pi_{1}\left(\mathfrak{o}_{L}\right) \longrightarrow \pi_{1}\left(\mathfrak{o}_{K}\right)
$$

is exact for pro-finite groups.

For example, let $L=\mathbb{Q}\left(\zeta_{p^{n}}\right)$ and $K=\mathbb{Q}$. Then there is a unique ramified point $\mathfrak{p} \mid p$ whose inertia coincides with the Galois group $G=\left(\mathbb{Z} / p^{n}\right)^{*}$. We choose $p, \mathfrak{p},(\mathfrak{p}, \mathfrak{p})$ as base points for $\operatorname{Spec}(\mathbb{Z}), \operatorname{Spec}\left(\mathbb{Z}\left[\zeta_{p^{n}}\right]\right), S^{\prime \prime}$. For the covering of $S^{\prime \prime}$ by its connected components the Seifert-Van Kampen Theorem gives

$$
\pi_{1}\left(S^{\prime \prime}\right)=\left(\underset{g \in G}{*} \pi_{1}\left(\operatorname{Spec}\left(\mathbb{Z}\left[\zeta_{p^{n}}\right], \mathfrak{p}\right)\right) / \text { identify all } \operatorname{Frob}_{\mathfrak{p}}\right.
$$

and consequently the exact sequence (5.15) yields a statement about the coinvariants:

$$
\left(\pi_{1}\left(\mathbb{Z}\left[\zeta_{p^{n}}\right]\right)\right)_{G\left(\mathbb{Q}\left(\zeta_{p^{n}}\right) / \mathbb{Q}\right)}=\pi_{1}(\mathbb{Z})=1 .
$$

Of course, thus also the coinvariants of the abelianised group which is the Galois group of the big Hilbert class field or also the coinvariants of the class group vanish.

Example 5.8. In [St89] a Seifert-Van Kampen theorem is applied to describe the unramified class field theory of an order in a number field. The corresponding effective descent map is the normalization map. The transfer to unramified class field theory is realized through abelianisation of fundamental groups. 


\section{§5.4. Examples from rigid/formal geometry}

Example 5.9. $\quad$ Let $k$ be a complete discrete valuation field and $S \subset$ $\mathbb{P}^{1}(k)$ a finite set of points. If the characteristic of $k$ is positive $\pi_{1}\left(\mathbb{P}^{1}-S\right)$ is still a mysterium. But also in the case of characteristic 0 the structure is only known by transcendental and ultimately topological means. In case $S$ has only two points and $n$ is invertible in $k$ the multiplication by $n$ yields a $\mathbb{Z} / n \mathbb{Z}$-Galois cover after identification of $\mathbb{P}^{1}-S$ with $\mathbb{G}_{m}$. Moreover, these covers are defined algebraically.

In [P92] Pop glues such covers in the rigid geometric category for suitable $S$ that are partitioned in pairs. A hypothesis on the distances of the points in $S$ allows to construct a big and algebraically defined quotient of $\pi_{1}\left(\mathbb{P}_{k^{\text {alg }}}^{1}-S\right)$ and furthermore control the action of $\operatorname{Gal}\left(k^{\text {alg }} / k\right)$. The glueing part can be understood by our treatment of the Seifert-Van Kampen theorem.

Example 5.10. In geometric Galois theory the notion of patching plays a fundamental role. It is used for example in the proof of the Abhyankar conjecture by Raynaud and Harbater or in attacks on the inverse Galois problem. Roughly speaking patching provides effective descent results in a formal or rigid geometric setting. For details we refer to [Ha03].

\section{§5.5. Examples from logarithmic geometry}

The notion of a Kummer étale cover in logarithmic geometry generalizes in the most convenient way the notion of tame ramification.

Example 5.11. A Seifert-Van Kampen theorem was apparently applied in [Saï97] Thm 2.8 to describe the Kummerian fundamental group of a semistable curve as the fundamental group of a graph of groups. Note that in this case the responsible 2-complex has no faces.

Example 5.12. An analogue of Theorem 5.2 in the logarithmic category was established in [Sx02] Thm I 3.2.25, Cor I 3.2.21. It is later on applied in [Sx02] to derive a version of Example 5.11 including Galois action that ultimately leads to anabelian geometry in positive characteristic.

\section{§5.6. The original Seifert-Van Kampen theorem}

The original Seifert-Van Kampen theorem requires a refinement of the above for discrete groups acting on arbitrary (not necessarily finite) sets. But then with $\mathscr{F}=\operatorname{Rev}^{\text {top }}$ being the category of topological covering maps fibred above the category of topological spaces Corollary 5.4 yields the following. 
Example 5.13. Let $X=U_{1} \cup U_{2}$ be an open covering of a topological space such that $U_{1}, U_{2}$ and $U=U_{1} \cap U_{2}$ are path-connected. The map $U_{1} \amalg U_{2} \rightarrow X$ is an effective descent morphism for Rev ${ }^{\text {top }}$ composed of monomorphisms. When we choose a base point $* \in U$ simultaneously for $X, U_{1}, U_{2}, U$ all paths $\gamma_{\text {., }}$ and $\alpha$ vanish. The simplicial set for this descent situation has the interval as its geometric realization. Hence

$$
\pi_{1}^{\mathrm{top}}(X, *) \cong \pi_{1}^{\mathrm{top}}\left(U_{1}, *\right) * \pi_{1}^{\mathrm{top}}(U, *) \pi_{1}^{\mathrm{top}}\left(U_{2}, *\right) .
$$

\section{References}

[Al75] Althoen, St. C., A Van Kampen theorem, J. Pure Appl. Algebra, 6 (1975), 41-47.

[Br67] Brown, R., Groupoids and Van Kampen's theorem, Proc. London Math. Soc., 17 (1967), 385-401.

[BRS84] Brown, R. and Razak Salleh, A., A van Kampen theorem for unions of nonconnected spaces, Arch. Math., 42 (1984), 85-88.

[Co92] Corson, J. M., Complexes of groups, Proc. London Math. Soc., (3) 65 (1992), 199-224.

[Gi64] Giraud, J., Méthode de la descente, Bull. Soc. Math. France Mém., 2, 1964.

[Ha03] Harbater, D., Patching and Galois theory, Galois groups and fundamental groups, 313-424, MSRI Publications, 41, Cambridge Univ. Press, 2003.

[Le79] Leroy, O., Groupoïde fondamentale et théorème de Van Kampen en théorie des topos, Cahiers mathématiques Montpellier, 17, Univ. Sci. Tech. Languedoc, Montpellier, 1979.

[Ma73] Magid, A. R., A Van Kampen theorem for separable algebras, Proc. Amer. Math. Soc., 39 (1973), 483-488.

[P92] Pop, F., $\frac{1}{2}$ Riemann existence theorem with Galois action, Algebra and Number theory (Essen 1992), 193-218, de Gruyter, 1994.

[RZ00] Ribes, L. and Zalesskii, P., Profinite Groups, Ergebnisse der Mathematik und ihrer Grenzgebiete, 40, Springer, 2000.

[Saï97] Saïdi, M., Revêtements modérés et groupe fondamental de graphe de groupes, Compositio Math., 107 (1997), 319-338.

[Se80] Serre, J.-P., Trees, Springer, 1980.

[SGA 1] Grothendieck, A. and Raynaud, M., Revêtements Étales et Groupe Fondamental (SGA 1), Lecture Notes in Math., 224, Springer, 1971.

[St89] Stevenhagen, P., Unramified class field theory for orders, Trans. Amer. Math. Soc., 311 (1989), 483-500.

[ST34] Seifert, H. and Threlfall, W., Lehrbuch der Topologie, B. G. Teubner, Leipzig, 1934.

[Sx02] Stix, J., Projective anabelian curves in positive characteristic and descent theory for log étale covers, thesis, Bonner Mathematische Schriften, 354, Bonn, 2002.

[VK33] Van Kampen, E., On the connection between the fundamental groups of some related spaces, Amer. J. Math., 55 (1933), 261-267.

[We61] Weinzweig, A. I., The fundamental group of a union of spaces, Pacific J. Math., 11 (1961), 763-776.

[Zo02] Zoonekynd, V., Théorème de Van Kampen pour les champs algébriques, Ann. Math. Blaise Pascal, 9 (2002), 145-145. 